Relations industrielles

Industrial Relations

\title{
L'obligation de disponibilité du salarié au-delà de son temps et de son lieu de travail : les enseignements de la Cour suprême du Canada dans Association des juristes de justice c Canada (Procureur général) \\ The Obligation of the Employee to Be Available Outside of his/her Working Hours and Place of Work: The Teachings of the Supreme Court of Canada Decision in Association of Justice CounselvCanada (Attorney General)
}

\section{Guylaine Vallée}

Volume 73, numéro 3, été 2018

URI : https://id.erudit.org/iderudit/1053842ar

DOI : https://doi.org/10.7202/1053842ar

Aller au sommaire du numéro

\section{Éditeur(s)}

Département des relations industrielles de l'Université Laval

ISSN

0034-379X (imprimé)

1703-8138 (numérique)

Découvrir la revue

Citer cet article

Vallée, G. (2018). L’obligation de disponibilité du salarié au-delà de son temps et de son lieu de travail : les enseignements de la Cour suprême du Canada dans Association des juristes de justice c Canada (Procureur général). Relations industrielles / Industrial Relations, 73(3), 591-602.

https://doi.org/10.7202/1053842ar
Résumé de l'article

Un employeur peut-il imposer à ses employés des périodes de garde obligatoires pendant lesquelles ils doivent être joignables en tout temps afin de pouvoir se rendre au travail rapidement et être en état d'accomplir leur prestation de travail ? Dans un arrêt rendu en 2017, la Cour suprême du Canada estime qu'une telle politique ne constitue pas un exercice raisonnable des droits de direction de l'employeur, mais qu'elle ne porte pas atteinte au droit à la liberté des employés protégé par la Charte canadienne. La démarche utilisée par la Cour pour apprécier ce qu'est l'exercice raisonnable d'un droit de direction représente la principale retombée de cet arrêt. Toutefois, l'analyse de l'obligation de disponibilité sous l'angle de l'atteinte aux droits fondamentaux des employés reste à faire.
Tous droits réservés @ Département des relations industrielles de l’Université Laval, 2018
Ce document est protégé par la loi sur le droit d'auteur. L’utilisation des services d'Érudit (y compris la reproduction) est assujettie à sa politique d'utilisation que vous pouvez consulter en ligne.

https://apropos.erudit.org/fr/usagers/politique-dutilisation/ 


\section{RUBRIQUE}

\section{L'obligation de disponibilité du salarié} au-delà de son temps et de son lieu de travail : les enseignements de la Cour suprême du Canada dans Association des juristes de justice c Canada (Procureur général)

\section{Guylaine Vallée}

L'employeur peut-il exiger de ses salariés qu'ils soient disponibles pour répondre rapidement à une éventuelle demande de travail et, ainsi, contrôler leur conduite et limiter leurs activités à l'extérieur du lieu de travail et en dehors de leurs heures de travail? L'imposition d'une telle politique constitue-t-elle un exercice raisonnable de ses droits de direction? Représente-t-elle une atteinte au droit fondamental à la liberté des salariés? Telles sont les questions abordées par la Cour suprême du Canada (ci-après CSC) dans l'arrêt Association des juristes

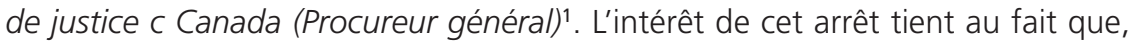
pour la première fois, la CSC traite d'une période, parfois qualifiée de «temps du troisième type » (Supiot, 1999: 123) ou de «troisième temps » (Vallée, 2010: 11), pendant laquelle les salariés, sans être réputés au travail, ne sont pas nécessairement en période de repos puisqu'ils ne peuvent vaquer librement à leurs activités personnelles, mais restent subordonnés à une éventuelle demande de travail de l'employeur. Cette période, qui brouille la frontière entre le temps de travail et le temps de repos établie dans la législation du travail et les conventions collectives, est un phénomène de plus en plus répandu en raison des nouvelles technologies des communications, des mutations de l'organisation du travail dans les entreprises et des transformations du travail (Boivin, 2016; Coiquaud, 2016; Genin, 2016; Vallée et Gesualdi-Fecteau, 2016; Vallée, 2016).

Les faits à l'origine de cette affaire concernent une directive par laquelle la Direction du droit de l'immigration du Bureau régional du Québec du ministère de la Justice Canada (ci-après l'employeur) impose, en 2010, des périodes de 
garde obligatoires et non rémunérées aux juristes à son emploi en dehors de leurs heures normales de travail. Compte tenu de la nature des activités de l'employeur, les juristes pouvaient être amenés à traiter de demandes urgentes de sursis après leur journée de travail, les soirs de semaine ou les fins de semaine. De 1990 à 2010, le système de garde fonctionnait sur une base volontaire: les juristes qui se portaient volontaires touchaient une contrepartie équivalant à 2,5 jours de congés payés pour une semaine de garde pendant les quarts du soir et de fin de semaine, à laquelle s'ajoutait une indemnité additionnelle pour les jours fériés. En 2010, l'employeur décide de mettre fin unilatéralement à cette contrepartie: les juristes ne seront, désormais, indemnisés que pour les heures de travail qu'ils accomplissent si leurs services sont requis pendant leur période de garde. Aucun juriste ne s'étant porté volontaire pour assurer des périodes de garde à la suite de ce changement, l'employeur décide d'imposer un système de garde obligatoire: tous les juristes devront, dorénavant, accomplir, en sus de leurs heures habituelles de travail, des quarts de garde de soir de $17 \mathrm{~h}$ à $21 \mathrm{~h}$ les jours de semaine et de $9 \mathrm{~h}$ à $21 \mathrm{~h}$ les fins de semaine, et ce, à raison d'une à trois semaines par année. Pendant ces périodes, les juristes doivent être joignables en tout temps, porter un téléavertisseur ou un téléphone cellulaire, pouvoir se rendre au travail dans un délai d'environ une heure s'ils sont appelés - ce qui limite leurs déplacements - et être en état d'accomplir leur prestation de travail. L'imposition par l'employeur de ce système de garde obligatoire est à l'origine du litige.

Les juristes en cause étant syndiqués et couverts par une convention collective, un grief fut déposé par I'Association des juristes de justice (ci-après AJJ). L'AJJ contestait l'imposition de la politique de garde obligatoire en se fondant sur deux arguments, qui ont déterminé les questions examinées par l'arbitre de la Commission des relations de travail et de l'emploi dans la fonction publique saisi du grief ${ }^{2}$, par la Cour d'appel fédérale (ci-après CAF) ${ }^{3}$ saisie d'une demande de contrôle judiciaire de la décision de l'arbitre et par la Cour suprême du Canada saisie d'un appel de la décision de la CAF. D'une part, l'AJJ prétendait qu'en imposant unilatéralement des périodes de garde obligatoires, l'employeur n'avait pas exercé ses droits de direction de manière raisonnable et équitable au sens de la convention collective. D'autre part, I'AJJ soutenait que ces périodes de garde obligatoires portaient atteinte au droit fondamental des juristes à la liberté au sens de l'article 7 de la Charte canadienne des droits et libertés ${ }^{4}$.

2 Association des juristes de justice et Conseil du trésor (ministère de la Justice), 2015 CRTEFP 31 [AJJ CRTEFP].

3 Procureur général du Canada et Association des juristes de justice, 2016 CAF 92 [AJJ CAF].

4 Charte canadienne des droits et libertés, partie I de la Loi constitutionnelle de 1982, constituant l'annexe B de la Loi de 1982 sur le Canada (R-U), 1982, c 11 [Charte canadienne]. 


\section{L'imposition d'une obligation de disponibilité: un exercice raisonnable des droits de direction de l'employeur?}

Sur cette première question, la CSC est divisée: si les sept juges de la majorité, dont les motifs sont rédigés par la juge Karakatsanis, et les deux juges de la minorité, sous la plume de la juge Côté, s'entendent sur l'identification de la

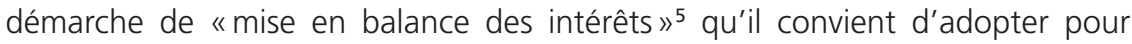
apprécier l'exercice raisonnable et équitable des droits de direction de l'employeur, ils sont en désaccord sur l'un des critères à considérer dans cette démarche et sur l'application qu'en a fait l'arbitre. Après avoir présenté la démarche retenue par la CSC dans l'arrêt AJJ (A), nous exposerons les incidences qu'il pourrait avoir sur l'appréciation de ce qu'est l'exercice raisonnable des droits de direction de l'employeur dans différents contextes (B).

A. La convention collective conclue entre l'employeur et I'AJJ était muette concernant I'obligation de disponibilité des juristes. Elle comportait une clause reconnaissant les droits résiduaires de direction de l'employeur, en vertu de laquelle l'employeur conservait ses droits de direction sur des matières non traitées par la convention collective. Ces droits résiduaires n'étaient pas absolus, la convention collective prévoyant notamment que l'employeur devait «agir raisonnablement, équitablement et de bonne foi dans l'administration » de la convention collective et, par conséquent, dans l'exercice des droits résiduaires que la convention lui reconnaissait ${ }^{6}$.

L'arbitre avait conclu que la directive de l'employeur n'était ni raisonnable ni équitable et il avait, en conséquence, ordonné à l'employeur de cesser de l'appliquer; l'absence de clauses sur l'obligation de disponibilité dans la convention collective ne faisait pas en sorte que l'employeur avait carte blanche pour imposer une période de garde et régir la conduite des salariés à l'extérieur du lieu de travail et au-delà des heures de travail, même si cette politique visait à satisfaire un besoin organisationnel. Contrairement à la CAF, la Cour suprême considère, à la majorité, que cette conclusion de l'arbitre est raisonnable et doit être maintenue, I'arbitre ayant appliqué la démarche de mise en balance des intérêts pour en arriver à ce résultat.

La démarche de mise en balance des intérêts, issue d'une jurisprudence arbitrale constante établie dans les provinces de common law, a été consacrée par la CSC dans l'arrêt Pâtes et Papier Irving7 rendu en 2013, dans lequel il s'agissait de déterminer si l'imposition unilatérale d'une politique de tests aléatoires obligatoires de dépistage d'alcool relevait d'un exercice raisonnable des droits de direction

5 Arrêt AJJ, supra note 1 aux para 24 (juge Karakatsanis) et 76 (juge Côté).

6 Clause 5.02 de la convention collective, dans Ibid au para 10.

7 Syndicat canadien des communications, de l'énergie et du papier, section locale 30 c Pâtes et Papier Irving, 2013 CSC 34 [Irving]. 
de l'employeur. Bien que l'arbitre n'ait pas explicitement cité l'arrêt Irving dans sa décision, la juge Karakatsanis estime qu'il a, néanmoins, appliqué une démarche basée sur la mise en balance des intérêts en appréciant, d'une part, le cadre de travail des juristes et l'effet de la directive de l'employeur sur leur vie personnelle et, d'autre part, l'objectif de l'employeur et le moyen qu'il a choisi pour l'atteindre $^{8}$. Ainsi, l'arbitre a tenu compte du cadre de travail des juristes, notamment en soulignant que leurs descriptions de tâches et leurs contrats d'emploi ne faisaient aucunement mention d'obligations de disponibilité ${ }^{\text {. }}$ Il a, de plus, examiné l'effet de la directive sur la vie des juristes, directive qui prolongeait le contrôle que l'employeur exerçait sur eux en dehors du travail d'une manière qui n'était pas prévue dans leurs contrats d'emploi ou leur convention collective, et tenu compte des circonstances entourant le changement unilatéral de la politique qui existait auparavant en matière de garde ${ }^{10}$. L'arbitre a aussi examiné l'objectif de l'employeur, qui était de combler un besoin organisationnel en matière d'urgence, même si la juge Karakatsanis souligne que les motifs de l'arbitre auraient pu être plus clairs ${ }^{11}$. Enfin, concernant le moyen choisi par l'employeur, l'arbitre a pris en considération le fait que l'employeur n'avait pas démontré que l'imposition de périodes de garde obligatoires était le seul moyen de satisfaire ce besoin. Sur cette question, la juge Karakatsanis rappelle que même si l'employeur n'avait pas à démontrer l'absence d'autres moyens pour atteindre son objectif, il reste que «l'existence de moyens réalistes, mais moins attentatoires, pour répondre aux besoins organisationnels peut constituer une considération pertinente dans l'appréciation axée sur la mise en balance des intérêts, conjointement avec la nature des intérêts de l'employeur et l'incidence de la politique sur les employés $»^{12}$. II s'agit du principal point de discorde entre les juges de la majorité et ceux de la minorité en ce qui concerne la démarche de mise en balance des intérêts appliquée par l'arbitre. La juge Côté, au nom des juges minoritaires, estime que, dans une telle démarche, le moyen choisi par l'employeur sera raisonnable si la nécessité de l'adopter pour atteindre son objectif l'emporte sur l'incidence négative qu'il a sur les salariés. En prenant en considération d'autres moyens, la juge Côté estime que l'arbitre a introduit un critère qui ne fait pas partie de la démarche de mise en balance des intérêts établie par la jurisprudence : il a commis une erreur dans l'appréciation du droit qui aurait justifié l'annulation de sa décision ${ }^{13}$.

8 Arrêt AJJ, supra note 1 au para 25.

9 Ibid au para 34.

10 Ibid aux para 35-36, 45-46.

11 Ibid aux para 41-42.

12 Ibid au para 44.

13 Ibid aux para 71-80. Outre cette erreur de droit, les juges de la minorité estiment que l'arbitre a commis des erreurs au regard des faits en passant sous silence des éléments de preuve 
B. Même si l'arrêt AJJ concerne une relation d'emploi dans la fonction publique fédérale, la démarche de mise en balance des intérêts utilisée pour évaluer I'exercice raisonnable et équitable des droits de direction de l'employeur au sens de la convention collective est susceptible d'avoir une influence dans d'autres contextes. En effet, les clauses de droit de direction interprétées dans cette affaire sont similaires à celles que l'on peut retrouver dans les conventions collectives des secteurs publics ou privés au Canada (Vallée et Bourgault, 2018: 91).

L'apport de la CSC, par ses arrêts AJJ et Irving rendus à quelques années d'intervalle, est aussi de proposer une démarche d'évaluation de ce qu'est l'exercice raisonnable d'un droit de direction. Cela est d'autant plus important que ce critère de "raisonnabilité » est de plus en plus utilisé pour apprécier l'exercice des droits de direction, et ce, même lorsque la convention collective ne le mentionne pas explicitement. Ainsi, le caractère raisonnable d'une politique adoptée unilatéralement par l'employeur en vertu de ses droits résiduaires est l'un des critères utilisés dans la jurisprudence pour juger de la validité d'une telle politique, et ce, tant dans les provinces de common law qu'au Québec ${ }^{14}$. De plus, au Québec, il est maintenant reconnu que les droits de direction de l'employeur ne peuvent être exercés d'une manière excessive et déraisonnable allant à l'encontre des exigences de la bonne foi ${ }^{15}$. Ces principes participent «de l'ordre public dans le contexte du contrat de travail ${ }^{16}$ et s'imposent à toute convention collective en vertu d'une jurisprudence constante ${ }^{17}$.

Enfin, il faut noter que dans l'arrêt AJJ, la CSC applique la démarche axée sur la «mise en balance» ou la «pondération» des intérêts à une politique portant sur des périodes de garde obligatoires alors que cette démarche semblait jusque-là utilisée pour évaluer le caractère raisonnable de politiques imposant des

sur la fréquence annuelle des périodes de garde, la prise en compte de la disponibilité des juristes pour établir ces périodes et la possibilité des juristes de s'accommoder entre eux (Ibid aux para 68-70) et en allant à l'encontre de la preuve sur les besoins organisationnels de I'employeur à l'origine de l'imposition unilatérale des périodes de garde (Ibid aux para 65$67,70)$.

14 KVP Co c Lumber and Sawmill Workers' Union, Local 2537, [1965] 16 LAC 73 [KVP], reprise dans Irving, supra note 7 aux para 24-26, Shell Canada Ltée et Travailleurs unis du pétrole du Canada, section locale 121 du SCEP, [2010] RJDT 247 (QCTA) aux para 44-46 [Shell]; Syndicat des métallos, section locale 7493 et Poudres métalliques du Québec Ltée, [2011] RJDT 879 (QCTA) aux para 92, 129 [Poudres métalliques].

15 Code civil du Québec, RLRQ c CCQ-1991, art 6-7.

16 Syndicat de l'enseignement de la région de Québec c Ménard, 2005 QCCA 440 au para 49.

17 Voir par exemple, sur le temps supplémentaire obligatoire des infirmières: Syndicat interprofessionnel du CHU de Québec (FIQ) et le Centre hospitalier universitaire de Québec, 2014 QCTA 836. 
tests de dépistage d'alcool ou de drogues ${ }^{18}$. On peut penser que la jurisprudence recourra à cette démarche pour évaluer le caractère raisonnable de politiques de l'employeur portant sur d'autres sujets.

\section{L'imposition d'une obligation de disponibilité: une atteinte aux droits fondamentaux des salariés?}

La convention collective entre l'employeur et I'AJJ prévoyait une autre limite aux droits de direction de l'employeur: celui-ci ne pouvait restreindre les droits constitutionnels des juristes ou les droits qui leur sont accordés par une loi fédérale ${ }^{19}$. L'AJJ estimait que la politique sur les gardes obligatoires de l'employeur constituait une atteinte au droit constitutionnel à la liberté des juristes garanti par l'article 7 de la Charte canadienne parce qu'elle limitait les choix qu'ils pouvaient faire dans leur vie privée. Parmi tous les décideurs saisis de cette affaire, seul l'arbitre a jugé que cet argument de l'AJJ était fondé. Même si la CAF et la CSC, à l'unanimitézo, considèrent que l'arbitre a commis une erreur dans l'interprétation de l'article 7 de la Charte canadienne et que sa décision portant sur ce motif doit être annulée (A), nous sommes d'avis que l'analyse de l'obligation de disponibilité sous l'angle de l'atteinte injustifiée aux droits fondamentaux des salariés reste pertinente, en particulier dans le contexte de la Charte des droits et libertés de la personne ${ }^{21}$ (B).

A. Dans l'arrêt AJ, la juge Karakatsanis écrit, au nom de la majorité, que «l'arbitre a, de toute évidence, surestimé la portée du droit à la liberté protégé par l'art. 7 »de la Charte canadienne ${ }^{22}$. L'arbitre avait considéré que cet article incluait «le droit de jouir d'une vie privée en dehors du lieu de travail et des heures normales de travail » et que la directive de l'employeur avait « des effets intrusifs sur l'intégrité de la cellule familiale et sur les responsabilités parentales des juristes» qui violaient leur droit à la vie privée ${ }^{23}$. La juge Karakatsanis rappelle que cet article ne protège pas «toutes les activités qu'une personne définit comme essentielles à son mode de vie ${ }^{24}$, mais bien les choix personnels fondamentaux «participant de l'essence même de ce que signifie la jouissance de la dignité et

18 Canadian National Railway Co and CAW, (2000) 95 LAC (4th) 341; Imperial Oil Ltd and Communications, Energy and Paperworkers Union of Canada, local 900, (2006) 157 LAC (4th) 225; citées et utilisées dans Irving, supra note 7 aux para 30-37 (juge Abella, pour la majorité); Shell, supra note 14 aux para 49-55; Poudres métalliques, supra note 14 aux para 99-104, 133.

19 Clause 6.01 de la convention collective, dans Arrêt AJJ, supra note 1 au para 11.

20 Ibid aux para 48-52 (juge Karakatsanis) et 54 (juge Côté).

21 RLRQ c C-12 [Charte québécoise].

22 Arrêt AJJ, supra note 1 au para 49.

23 AJJ CRTEFP, supra note 2 au para 62.

24 Arrêt AJJ, supra note 1 au para 50. 
de l'indépendance individuelle » ${ }^{25}$. Or, les activités des juristes qui se trouvaient affectées par les périodes de garde obligatoires, comme assister aux spectacles compris dans un abonnement ou recevoir des amis le week-end, ne sont pas protégées par l'article 7 de la Charte canadienne. Même les activités parentales ou familiales que les juristes ne pouvaient accomplir lorsqu'ils étaient en période de garde, comme conduire leurs enfants au hockey ou visiter des membres de leur famille lorsque cela faisait en sorte qu'ils se trouvaient à plus d'une heure des lieux de travail, ne sont pas protégées par l'article 7 de la Charte canadienne ${ }^{26}$. La CAF avait, pour sa part, noté que cet argument aurait été plus convaincant si les périodes de garde avaient été plus fréquentes ${ }^{27}$.

B. Même si tous les juges de la CSC ont considéré qu'en l'espèce, la politique de l'employeur ne violait pas le droit à la liberté des juristes, nous sommes d'avis que I'arrêt AJJ ne tranche pas de manière définitive la question de savoir si l'imposition de périodes de disponibilité obligatoires porte atteinte aux droits fondamentaux des salariés. En effet, la seule source que pouvait mobiliser l'AJJ au soutien de son argument était l'article 7 de la Charte canadienne ${ }^{28}$. Or, il n'est pas clairement établi, comme le rappelle la CSC, que cet article s'applique en dehors du contexte de l'administration de la justice ${ }^{29}$. Cette incertitude peut expliquer la prudence de la CSC dans son interprétation de l'article 7 dans l'affaire en cause.

Cette question pourrait être traitée différemment en vertu de la Charte québécoise. Contrairement à la Charte canadienne, la Charte québécoise s'applique, tant dans les secteurs privé que public, aux relations individuelles ou collectives de travail qui relèvent de la compétence législative du Québec. La Charte québécoise protège notamment le droit au respect de la vie privée ${ }^{30}$, le droit à la liberté et à

25 Ibid au para 49 [références omises].

26 Ibid au para 51.

27 AJJ CAF, supra note 3 au para 41.

28 La Loi canadienne sur les droits de la personne [LRC 1985, c H-6], applicable aux entreprises de juridiction fédérale, a pour objet d'interdire la discrimination pour un motif illicite et ne reconnaît pas les droits fondamentaux comme le droit à la liberté ou à la vie privée.

29 Arrêt AJJ, supra note 1 au para 49. Notons, toutefois, que l'article 7 de la Charte canadienne a déjà été utilisé pour déclarer inopérante la clause d'une entente collective qui établissait des périodes de garde de 24 heures pour les médecins résidents d'un Centre universitaire de santé : Centre universitaire de santé McGill et Association des résidents de McGill, [2011] RJDT 853 (QCTA) aux para 134-157, requête en révision judiciaire, QCCS, le 7 juillet 2011 [Association des résidents]. L'arbitre a considéré que la Charte canadienne était applicable au litige [Ibid aux para 136-139]; que les périodes de garde portaient atteinte au droit à la sécurité des médecins et de leurs patients et que cette atteinte n'était pas conforme aux principes de justice fondamentale comme l'exige l'article 7 de la Charte canadienne [Ibid aux para 140-148]; et que cette atteinte n'était pas justifiée au sens de l'article 1 de la Charte canadienne [Ibid aux para 149-157].

30 Charte québécoise, art 5. 
I'intégrité ${ }^{31}$ ainsi que le droit à des conditions de travail justes et raisonnables ${ }^{32}$. L'imposition de périodes de disponibilité obligatoires pourrait être examinée sous l'angle de l'atteinte à ces droits fondamentaux.

Le fait qu'une personne se voit contrainte, en dehors de son temps de travail, d'être joignable, disponible pour travailler et en état de le faire si l'employeur le lui demande pourrait porter atteinte à son droit au respect de la vie privée garanti par l'article 5 de la Charte québécoise. Ce droit protège notamment le droit d'une personne "à l'autonomie dans l'aménagement de sa vie personnelle et familiale »33, «le droit d'être laissé tranquille » ou « une sphère de chaque existence dans laquelle nul ne peut s'immiscer sans y être convié» (Potvin, 1991: 156-157). Des politiques imposant une obligation de résidence ${ }^{34}$ ou le travail à domicile ${ }^{35}$ ont été considérées comme des atteintes au droit à la vie privée des salariés. Comme les autres droits et libertés fondamentaux protégés par la Charte québécoise, le droit au respect de la vie privée n'est toutefois pas absolu ${ }^{36}$. En vertu de l'article 9.1 de la Charte québécoise, I'atteinte à une liberté ou à un droit fondamental peut être justifiée si l'employeur démontre que l'objectif qu'il poursuit est légitime et important, et que le moyen choisi est proportionnel à cet objectif, c'est-à-dire qu'il est rationnellement lié à celui-ci et que l'atteinte au droit est minimale ${ }^{37}$. Par exemple, en vertu de ces critères, une politique instaurant des périodes de disponibilité obligatoires pourrait, même si elle était considérée comme une atteinte au droit à la vie privée des salariés, être justifiée si l'employeur faisait la preuve qu'elle répond à un objectif légitime et important, par exemple assurer des services essentiels devant être garantis en tout temps, et qu'elle est proportionnelle à cet objectif. II lui faudrait alors démontrer qu'il existe un lien rationnel entre sa politique instaurant des périodes de disponibilité obligatoires et l'objectif légitime et important, et que cette politique est le moyen d'atteindre l'objectif qui porte le moins atteinte au droit à la vie privée des salariés [atteinte minimale].

$31 \mathrm{lbid}$, art 1.

$32 \mathrm{lbid}$, art 46.

33 The Gazette (Division Southam inc.) c Valiquette, 1996 CanLII 6064 (QCCA) à la p 10; repris dans Godbout c Ville de Longueuil, [1997] 3 RCS 844 au para 98 [Godbout]; Syndicat des professionnelles du Centre jeunesse de Québec (CSN) c Desnoyers, 2005 QCCA 110 au para 26 [Desnoyers].

34 Godbout, supra note 33.

35 Desnoyers, supra note 33.

36 Charte québécoise, art 9.1.

37 Rappelons que le test de justification fondé sur l'article 1 de la Charte canadienne élaboré dans I'arrêt Oakes [R c Oakes, [1986] 1 RCS 103 aux para 68-71] est applicable à l'article 9.1 de la Charte québécoise: Ford c Québec (Procureur général), [1988] 2 RCS 712 aux para 62-63; Godbout, supra note 33 aux para 3, 103-104. 
Des périodes de garde prolongées pourraient aussi porter atteinte au droit à I'intégrité garanti par l'article 1 de la Charte québécoise. Dans l'affaire Association des résidents ${ }^{38}$, un arbitre a conclu que la clause d'une entente collective qui établissait des périodes de garde de 24 heures pour les médecins résidents d'un Centre universitaire de santé portait atteinte au droit à l'intégrité des médecins résidents et de leurs patients. La preuve avait démontré que ces périodes de garde affectaient la santé physique ou psychique des médecins et provoquaient un état de fatigue engendrant un risque accru d'erreurs médicales qui mettait en péril la santé des patients ${ }^{39}$. L'arbitre avait considéré que cette atteinte au droit à l'intégrité n'était pas justifiée au sens de l'article 9.1 de la Charte québécoise. L'employeur n'avait pas démontré que les objectifs qu'il poursuivait par ces périodes de garde de 24 heures - favoriser un meilleur apprentissage pour les résidents, réduire la durée de leur période de formation et engendrer, de ce fait, des économies budgétaires - étaient suffisamment importants pour justifier l'atteinte au droit à l'intégrité des résidents et des patients. Même en admettant que l'objectif d'un meilleur apprentissage était légitime et important, l'employeur n'avait pas fait la preuve que le moyen choisi, soit des périodes de garde de 24 heures, était rationnellement et proportionnellement relié à cet objectif. En particulier, l'employeur n'avait pas démontré en quoi une période de garde de 24 heures, plutôt qu'une période plus courte, favorisait un meilleur apprentissage des médecins résidents ${ }^{40}$.

Enfin, dans l'affaire Association des résidents, l'arbitre a aussi considéré que la clause de l'entente collective sur les périodes de garde de 24 heures des médecins résidents violait leur droit à des conditions de travail justes et raisonnables établi par l'article 46 de la Charte québécoise. Il lui "apparaît évident » ${ }^{41}$ qu'une condition de travail comportant des risques de blessures pour les médecins résidents et leurs patients est injuste et déraisonnable ${ }^{42}$.

\section{Conclusion}

L'obligation de disponibilité des salariés se manifeste de plusieurs manières dans les milieux de travail: elle peut être imposée, avec ou sans contreparties, pour des périodes de garde bien délimitées dans le temps, comme dans l'arrêt $A J$, ou encore pour des périodes beaucoup plus longues et moins bien balisées, ce qui augmente encore le contrôle que l'employeur peut avoir sur la vie personnelle du salarié (Vallée et Gesualdi-Fecteau, 2017: 272-276). Les règles relatives

38 Association des résidents, supra note 29.

39 Ibid aux para 131-133, 161-163.

40 Ibid au para 163, renvoyant aux para 149-157.

41 Ibid au para 168.

42 Ibid aux para 164-169. 
à la durée du travail que l'on retrouve dans la législation du travail ou dans les conventions collectives ne parviennent pas à capter la nature particulière de ces périodes obligatoires de disponibilité. Celles-ci sont parfois considérées comme un temps de travail ouvrant droit à rémunération et prises en compte aux fins du calcul de la semaine normale de travail; dans d'autres cas, elles sont considérées comme du temps de repos, en dépit des limites qu'elles imposent aux activités du salarié (Vallée et Gesualdi-Fecteau, 2017: 277-289; Vallée, 2010: 11-15).

Deux moyens de contester l'imposition de telles obligations de disponibilité par l'employeur ont été examinés par la CSC dans l'arrêt Association des juristes de justice c Canada (procureur général), ainsi que nous l'avons vu. II y a une similitude certaine entre ces moyens qui exigent de procéder à une forme de pondération pour déterminer si les effets négatifs de l'obligation pour les salariés l'emportent sur ses effets bénéfiques pour l'employeur ${ }^{43}$. Toutefois, lorsqu'il y a atteinte à un droit fondamental, le fardeau de l'employeur est plus lourd puisqu'il doit notamment démontrer, dans le cadre du test de justification fondé sur l'article 1 de la Charte canadienne ou sur l'article 9.1 de la Charte québécoise ${ }^{44}$, que la politique qu'il a imposée représente une atteinte minimale aux droits fondamentaux du salarié ${ }^{45}$. II reste que, quel que soit le moyen choisi, il n'aura d'effet immédiat que pour les salariés ou les syndicats qui ont contesté de telles obligations de disponibilité devant un tribunal.

Un autre moyen, législatif celui-là, serait de reconnaître au salarié, en sus du droit de refuser de travailler au-delà d'un certain seuil ${ }^{46}$, le droit de ne pas être disponible pour certaines périodes ou au-delà d'un certain seuil sur une base hebdomadaire. Par exemple, le Code du travail français reconnaît un «droit à la déconnexion» passant par «la mise en place par l'entreprise de dispositifs de régulation de l'utilisation des outils numériques, en vue d'assurer le respect des temps de repos et de congé ainsi que de la vie personnelle et familiale ${ }^{47}$ et définit les périodes d'astreinte donnant droit à une contrepartie sous forme

43 Poudres métalliques, supra note 14 aux para 127, 132. Voir aussi : Section locale 143 du Syndicat canadien des communications, de l'énergie et du papier c Goodyear Canada Inc., 2007 QCCA 1686 aux para 19, 33-34; Shell, supra note 14 aux para 47-55.

44 Voir la note 37 et le texte correspondant.

45 Shell, supra note 14 au para 55. Plaidoyer en faveur d'une prise en compte du test de proportionnalité en vertu des Chartes dans la démarche de mise en balance des intérêts : Alon-Shenker et Davidov, 2013 : 391-393, 406-419.

46 Loi sur les normes du travail, RLRQ c N-1.1, art 59.0.1.

47 Code du travail (France), version consolidée au 15 avril 2018, art L2242-17 (7), tel que modifié par l'art 7 de l'ordonnance n 2017-1385 du 22 septembre 2017, en ligne : https:// www.legifrance.gouv. fr/affichCode.do?cidTexte $=$ LEGITEXT000006072050\&dateTexte $=201$ 80421 (consulté le 20 avril 2018). Voir aussi, au Québec, le Projet de loi 1097, Loi sur le droit à la déconnexion, $1^{\text {er }}$ session, 41 le législature, Québec, 2018 (présenté le 22 mars 2018). 
financière ou sous forme de repos comme les périodes pendant lesquelles «le salarié, sans être sur son lieu de travail et sans être à la disposition permanente et immédiate de l'employeur, doit être en mesure d'intervenir pour accomplir un travail au service de l'entreprise $~^{48}$. La définition de normes minimales applicables à l'ensemble des salariés permettrait d'encadrer, pour tous, ces pratiques au cœur des transformations du travail.

\section{Références bibliographiques}

Alon-Shenker, Pnina et Guy Davidov (2013) «Applying the Principle of Proportionality in Employment and Labour Law Contexts », McGill Law Journal, 59 (2), 375-423.

Boivin, Louise (2016) «'Just-in-Time' Labour: The Case of Networks Providing Home Support Services in Quebec», International Journal of Comparative Labour Law and Industrial Relations, 32 (3), 301-321.

Coiquaud, Urwana (2016) "The Obligation to Be Available: The Case of the Trucking Industry », International Journal of Comparative Labour Law and Industrial Relations, 32 (3), 322-343.

Genin, Émilie (2016) «Proposal for a Theoretical Framework for the Analysis of Time Porosity », International Journal of Comparative Labour Law and Industrial Relations, 32 (3), 280-300.

Potvin, Louise (1991) La personne et la protection de son image. Étude comparée des droits québécois, français et de common law anglaise, Cowansville: Éditions Yvon Blais, 523 pages.

Supiot, Alain, dir. (1999) Au-delà de l'emploi, Paris: Flammarion, 321 pages.

Vallée, Guylaine (2010) "Les nouvelles formes d'emploi et le "brouillage" de la frontière entre la vie de travail et la vie privée: jusqu'où va l'obligation de disponibilité des salariés?», Lex Electronica, 15 (2), 1-34, en ligne: <www.lex-electronica.org/files/sites/103/15-2_vallee. $\mathrm{pdf}>$.

Vallée, Guylaine (2016) «Employees' Obligation to Be Available to Employers: A (New) Pathway to Precariousness or a Source of Flexibility? [Guest Editorial]», International Journal of Comparative Labour Law and Industrial Relations, 32 (3), 275-279.

Vallée, Guylaine et Dalia Gesualdi Fecteau (2016) «Setting the Temporal Boundaries of Work: An Empirical Study of the Nature and Scope of Labour Law Protections », International Journal of Comparative Labour Law and Industrial Relations, 32 (3), 344-378.

Vallée, Guylaine et Dalia Gesualdi-Fecteau (2017) «Le travail à la demande et I'obligation de disponibilité des personnes salariées: portée des balises fixées par la Loi sur les normes du travail», Service de la formation continue, Barreau du Québec, vol. 429, Développements récents en droit du travail, Montréal : Éditions Yvon Blais, 257-297.

Vallée, Guylaine et Julie Bourgault (2018), « Droits de direction », dans P. Jalette, M. Laroche et G. Trudeau (dir.), La convention collective au Québec, 3e éd., Montréal: Chenelière Éducation, 91-110.

48 Code du travail (France), supra note 47, art L3121-9 (1) et (3), tel que modifié par l'art 8 de la Loi no 2016-1088 du 8 août 2016. 


\section{RÉSUMÉ}

\section{L'obligation de disponibilité du salarié au-delà de son temps et de son lieu de travail: les enseignements de la décision de la Cour suprême du Canada dans Association des juristes de justice c Canada (Procureur général)}

Un employeur peut-il imposer à ses employés des périodes de garde obligatoires pendant lesquelles ils doivent être joignables en tout temps afin de pouvoir se rendre au travail rapidement et être en état d'accomplir leur prestation de travail? Dans un arrêt rendu en 2017, la Cour suprême du Canada estime qu'une telle politique ne constitue pas un exercice raisonnable des droits de direction de l'employeur, mais qu'elle ne porte pas atteinte au droit à la liberté des employés protégé par la Charte canadienne. La démarche utilisée par la Cour pour apprécier ce qu'est l'exercice raisonnable d'un droit de direction représente la principale retombée de cet arrêt. Toutefois, I'analyse de l'obligation de disponibilité sous l'angle de l'atteinte aux droits fondamentaux des employés reste à faire.

MOTS-CLÉS: obligation de disponibilité, période de garde, exercice raisonnable des droits de direction, droits fondamentaux des employés.

\section{SUMMARY}

The Obligation of the Employee to Be Available Outside of his/ her Working Hours and Place of Work: The Teachings of the Supreme Court of Canada Decision in Association of Justice Counsel v Canada (Attorney General)

Can an employer impose mandatory on-call periods on its employees during which they must be reachable at all times in order to be able to get to work quickly and perform their work? In a judgment rendered in 2017, the Supreme Court of Canada considered that such a policy does not constitute a reasonable exercise of an employer's management rights, but that it does not affect the employees' freedom of rights protected by the Canadian Charter. The approach taken by the Court in assessing what constitutes the reasonable exercise of management rights is the main outcome of this judgment. However, the analysis of the obligation of availability in terms of the violation of employees' fundamental rights remains to be done.

KEYWORDS: obligation of availability, on-call period, reasonable exercise of management rights, fundamental rights of employees. 